\title{
¿QUÉ TERAPIAS PSICOLÓGICAS SON EFICACES? UN RETO ANTE EL AÑO 2000
}

\author{
ENRIQUE ECHEBURÚA \\ Universidad del País Vasco \\ (Recibido el 18 de septiembre de 1998)
}

\begin{abstract}
Las terapias psicológicas han experimentado un gran desarrollo en diferentes áreas. En este artículo se describe una lista de los tratamientos de trastornos específicos que cuentan con un apoyo empírico. Los diferentes enfoques terapéuticos valiosos tienen en común el énfasis en la solución de problemas. En los tratamientos con muchos componentes es preciso depurar los componentes efectivos de los que no lo son. Se analizan también los últimos desarrollos y las tendencias de futuro en este ámbito.
\end{abstract}

Palabras clave: Tratamientos psicológicos, terapias validadas empíricamente, efectividad.

Which psychological treatments are effective? A challenge in front of the millennium

Psychological treatments are maturing on a broad front. In this paper a list of empirically supported psychological treatments for specific target populations is described. Much psychological treatment can be construed as problem-solving, a theme common to many apparently different approaches. In the multicomponent treatments it is necessary to carry out a fractionated distillation of those therapeutic ingredients that are essential and those that are redundant. Recent developments and possible future trends are considered.

Key words: Psychological treatments, empirically validated therapies, effectiveness.

\section{INTRODUCCIÓN}

La psicología clínica ha evolucionado en pocos años del mero psicodiagnóstico a la aplicación sistemática de tratamientos psicológicos. De este modo, la psicología clínica actual se configura como una disciplina que describe los trastornos psicológicos, genera unas técnicas de intervención y se articula en una red asistencial determinada.

La demostración experimental de la eficacia de los procedimientos terapéuticos se ha convertido actualmente en un objetivo prioritario. Las razones son

Parte de este artículo fue presentado a la Jornada Monográfica sobre la Psicología Clínica, organizada por la Asociación Española de Psicología Clínica y Psicopatología, celebrada en Valencia el 28 de marzo de 1998. varias: a) la debilidad y la multiplicidad de los modelos teóricos, no ajenas al divorcio creciente entre el mundo académico y la realidad clínica; b) la demanda social de tratamientos eficaces; y c) el objetivo prioritario de los terapeutas de mejorar a los pacientes de la forma más efectiva. La coherencia inicial postulada entre la teoría y la práctica se ha debilitado a expensas de una atención selectiva a la utilidad de la intervención terapéutica. El énfasis actual en el carácter tecnológico de las terapias psicológicas ha llevado en muchos casos a minimizar el papel de la teoría. De hecho, la evi-

Correspondencia: Enrique Echeburúa. Universidad del País Vasco. Facultad de Psicología. Departamento de Personalidad, Evaluación y Tratamientos Psicológicos. Avda. de Tolosa, 70. 20009 San Sebastián. Correo-e: ptpodece@ss.ehu.es. 
dencia experimental se ha referido especialmente a la comprobación de la eficacia de las técnicas terapéuticas, pero no así a la verificación de los principios teóricos en que supuestamente se basan (Echeburúa, 1993).

El economicismo vigente en la Sanidad Pública - lo que ahora se denomina la eficiencia de los servicios-y en las Compañías de Seguros ha impulsado en los últimos años la investigación sobre la eficacia de las terapias psicológicas. El objetivo, en última instancia, es financiar sólo, al menos en el ámbito de los Centros de Salud Públicos, aquellos tratamientos que sean efectivos y eficientes (Barlow, 1996; Shapiro, 1996). No es casual, por ejemplo, que hayan desaparecido recientemente el psicoanálisis y la hipnosis de los servicios que presta la Seguridad Social en España.

Al margen de los posibles excesos cometidos por un enfoque meramente economicista de la salud, no cabe duda de que esta orientación ha supuesto - y va a suponer aún más - un giro radical en la investigación sobre los tratamientos psicológicos. Ya no se trata sólo de demostrar que una terapia es eficaz para un determinado trastorno, sino que lo es más que otras alternativas y en unas mejores condiciones (más breve, en un régimen ambulatorio, etc.). El corolario de todo ello es que las terapias van a tender a estar protocolizadas, es decir, con una descripción precisa de los instrumentos de evaluación, las sesiones de tratamiento, el contenido concreto de cada una de ellas, etc.

La preocupación actual por el incumplimiento de las prescripciones terapéuticas -un fenómeno mucho más frecuente de lo que habitualmente se señala - ha llevado a la adopción de tratamientos cada vez más sencillos. Así como la ingesta de psicofármacos se realiza según las pautas establecidas si el paciente entiende para qué sirven los fár- macos, si toma pocos y las instrucciones son simples (Marks, 1981), las terapias psicológicas deben ser sencillas y con no muchas tareas entre sesiones. En caso contrario, como ocurre con el entrenamiento en inoculación de estrés, la probabilidad de incumplimiento puede ser alta (Foa, Rothbaum, Riggs y Murdock, 1991).

No deja de resultar paradójico, sin embargo, que el establecimiento de un tratamiento simple venga siempre precedido de investigaciones complejas que permiten delimitarlo y depurarlo Marks, 1991).

\section{TRATAMIENTOS PSICOLÓGICOS OFRECIDOS}

En los últimos 10 años se han producido tres avances muy importantes en el campo de los trastornos mentales. En primer lugar, se han hecho grandes adelantos en la comprensión de las bases biológicas de muchos cuadros clínicos (la esquizofrenia, la depresión o los trastornos bipolares, por ejemplo). En segundo lugar, se ha desarrollado una nueva generación de psicofármacos (antidepresivos y neurolépticos especialmente), con mayor eficacia terapéutica y un perfil más favorable de efectos secundarios. $Y$ en tercer lugar - pero no menos importante--, se han creado tratamientos psicológicos breves y efectivos para una amplia variedad de trastornos.

La difusión de estos tres avances no ha sido simétrica. En los dos primeros casos las compañías farmacéuticas multinacionales se han ocupado de difundirlos ampliamente mediante diversas publicaciones gratuitas (folletos, revistas, libros, etc.) y congresos financiados. Sin embargo, en el tercer caso - el desarrollo de los tratamientos psicológicos-, al no contar con un órgano de difusión tan poderoso como las multinacionales farmacéuticas 
(que, por motivos obvios, no están interesadas en este tema), los avances espectaculares habidos se han limitado a las revistas científicas y no han llegado siquiera a los sectores profesionales implicados ni se les ha prestado la atención debida.

En estos momentos hay inventariadas más de 250 terapias psicológicas diferentes (Herink, 1980). En EE.UU. y en Europa florece actualmente una plétora de grupos de crecimiento, terapias gestálticas, seminarios de percepción extrasensorial, etc. Sin embargo, la sanidad pública sólo debe sufragar los tratamientos bien contrastados empíricamente, es decir, los más eficaces y, en igualdad de condiciones, los más breves. Acortar el sufrimiento del paciente y ahorrar gastos y tiempo a los terapeutas de los Centros de Salud Mental parecen objetivos prioritarios.

Es más, la evaluación no puede limitarse exclusivamente a la mayor o menor eficacia de una terapia sobre otra en un trastorno específico. En concreto, según un análisis de costes y beneficios, la evaluación debe centrarse también en los siguientes aspectos:

a) La evaluación del tratamiento ambulatorio o de asistencia domiciliaria en comparación con el llevado a cabo en régimen de internamiento (por ejemplo, en el ámbito del alcoholismo, de las toxicomanías o de la esquizofrenia).

b) La valoración de los tratamientos grupales en comparación con los individuales (por ejemplo, en el ámbito de los trastornos de ansiedad, adictivos o de la conducta alimentaria).

c) La evaluación de los programas de autoterapia o de intervención profesional mínima en comparación con el formato tradicional terapeuta-paciente. De este modo, han surgido en los últimos años protocolos específicos de actuación terapéutica, basados en los manuales de autoayuda (Becoña, 1993; Fernández-
Montalvo y Echeburúa, 1997; Foa y Wilson, 1992; Labrador, 1992; McCrady, Rodríguez y Otero, 1997; Sevillá y Pastor, 1996) o difundidos por la tecnología de la comunicación a distancia (teléfono, correo, TV, Internet, etc.) (Baer y Greist, 1997; Burgess, Gill y Marks, 1998; Greist, Marks, Baer et al., 1998; McKenzie, Blanes y Marks, 1995; Brengelmann, 1977; McAllister, 1976).

Sin embargo, las limitaciones de este enfoque -es decir, la pérdida del contacto entre paciente y terapeuta- se han puesto de relieve en otras investigaciones (por ejemplo, Hickling y Blanchard, 1997). Por otra parte, a excepción de unos pocos estudios (Salaberría y Echeburúa, 1998), la validez de la manuales de autoayuda no ha sido puesta a prueba.

d) La evaluación de la eficacia de otros profesionales (enfermeras psiquiátricas, trabajadores sociales, maestros, etc.) como terapeutas en sus medios profesionales (Ginsberg, Marks y Walters, 1984).

e) La evaluación de la eficacia de las estrategias de intervención mínima a cargo de los Equipos de Atención Primaria (cfr. Derogatis y Wise, 1996).

f) La valoración del grado de observancia de las prescripciones terapéuticas (Labrador y Castro, 1987).

Por último, la evaluación de los resultados de las terapias psicológicas (es decir, del grado de eficacia) no debe soslayar la necesidad de evaluar el proceso de las mismas: cómo funcionan y a qué tipo de factores se puede atribuir el cambio de comportamiento ( $c f r$. Caro, 1993; Hersen y Michelson, 1986).

No deja de ser significativo que de este tema - la evaluación de la eficacia de los tratamientos - se hayan ocupado muy pocos de los textos clínicos -entre ellos, Cottraux (1991), Echeburúa (1993) y Pérez (1996) - que versan sobre terapias psicológicas. 


\section{EVALUACIÓN DE LOS RESULTADOS}

En los cinco últimos años ha habido un intento riguroso —el más serio de los emprendidos hasta el momento- para evaluar la eficacia de las terapias psicológicas, hecho desde diversos frentes. $\mathrm{Ya}$ no se trata de determinar la validez de las psicoterapias en su conjunto o como aplicación a problemas psicológicos inespecíficos, como se hacía en las últimas décadas (por ejemplo, Smith, Glass y Miller, 1980), sino de evaluar tratamientos eficaces para trastornos concretos en muestras clínicas claramente especificadas (Barlow, 1994; Chambless y Hollon, 1998).

A iniciativa del Congreso de EE.UU., se ha creado recientemente una agencia federal -la Agency for Health Care Policy and Research-que tiene como objetivo determinar la eficacia de los tratamientos psicológicos para los trastornos mentales y establecer un directorio de las terapias de eficacia probada, con la finalidad última de mejorar la calidad del sistema de salud. Las guías elaboradas sirven, entre otras finalidades, como criterio de financiación para las Compañías Aseguradoras, en cuanto al tipo y duración de una terapia, y para resolver demandas judiciales en relación con una práctica profesional supuestamente inadecuada. Se trata asimismo de orientar a los usuarios y de dotar de criterios a los responsables de los Servicios de Salud Mental para promover en los Centros Públicos sólo aquellas terapias que estén validadas empíricamente (Chambless, 1996).

En la elaboración de este informe, auspiciado por la División 12 (Psicología Clínica) de la Asociación Psicológica Americana y dirigido por Chambless, han participado terapeutas de distintas orientaciones (psicodinámicos, interpersonales y cognitivo-conductuales) (Task Force on Promotion and Dissemination of Psychological Procedures, 1995).

La determinación de la eficacia de un tratamiento viene avalada por la presencia de tres criterios: a) estar respaldado por la existencia de dos o más estudios rigurosos de investigadores distintos con diseños experimentales intergrupales ( $N=30$ sujetos por grupo); b) contar con un manual de tratamiento claramente descrito; y c) haber sido puesto a prueba en una muestra de pacientes inequívocamente identificados (por ejemplo, con arreglo a los criterios diagnósticos del $D S M-I V$ ). De hecho, la taxonomía del $D S M-I V$ es un requisito cada vez más exigido en todos los ámbitos (forense, clínico, etc.) (Hickey, 1998).

Las condiciones necesarias para un ensayo clínico figuran agrupadas en la Tabla 1 (Seligman, 1995).

Tabla 1. Requisitos convenientes en las investigaciones clínicas sobre la eficacia de tratamientos (Seligman, 1995, modificado)

- Asignación aleatoria de los pacientes a las condiciones experimentales y de control. Mínimo de 30 sujetos por cada modalidad terapéutica.

- Evaluación detallada con arreglo a criterios diagnósticos operativos del DSM-IV. Medidas de evaluación múltiples.

- Entrevistadores ciegos respecto al grupo de tratamiento asignado al paciente evaluado.

- Exclusión de pacientes con trastornos múltiples.

- Tratamientos claramente descritos y protocolizados.

- Fijación de un número determinado de sesiones.

- Seguimiento de los pacientes a largo plazo. 
De modo complementario, Chambless y Hollon (1998) se han referido a la existencia de tres tipos de eficacia: a) tratamiento eficaz: aquel que es mejor que la ausencia de terapia en, al menos, dos estudios independientes; b) tratamiento probablemente eficaz: aquel que no ha sido replicado todavía; y c) tratamiento eficaz $y$ específico: aquel que es mejor que un tratamiento alternativo o que un placebo.

En las Tablas 2, 3 y 4 figura una lista actualizada de los tratamientos psicológicos apoyados empíricamente (Chambless, Sanderson, Shoham et al., 1996). Se trata de una verificación empírica (experimental) y no meramente clínica (subjetiva), con una referencia clara al coste/eficacia. De esta lista se pueden obtener varias conclusiones: a) la superioridad de las terapias cognitivo-conductuales como tratamientos de elección; b) la ausencia generalizada de las terapias psicodinámicas, a excepción de la terapia interpersonal para la depresión y la bulimia y quizá algunas intervenciones psicodinámicas breves. El tema no está, en modo alguno, cerrado. Una nueva generación de estudios debe centrarse en los fracasos del tratamiento, es decir, de qué modo predecirlos y qué alternativas terapéuticas plantear en esos casos (Wilson, 1996).

Al margen de las limitaciones del informe (por ejemplo, las diferencias de rendimiento de los terapeutas con una misma terapia, la variación de la gravedad de pacientes con un mismo diagnóstico, la inexistencia de pacientes "puros», etc.) (Garfield, 1996; Havik y VanderBoss,

Tabla 2. Tratamientos psicológicos efectivos (Chambless et al., 1996, modificado)

\begin{tabular}{|c|c|}
\hline TIPOS DE TRATAMIENTOS & EVIDENCIA EMPÍRICA \\
\hline \multicolumn{2}{|c|}{ Trastornos de ansiedad } \\
\hline Terapia cognitivo-conductual para el trastorno de pánico & $\begin{array}{l}\text { Barlow et al. (1989) } \\
\text { Clark et al. (1994) }\end{array}$ \\
\hline $\begin{array}{l}\text { Terapia de exposición para las fobias (específica, social y } \\
\text { agorafobia) y el trastorno de estrés postraumático }\end{array}$ & $\begin{array}{l}\text { Öst et al. (1991) } \\
\text { Heimberg et al. (1990) } \\
\text { Trull et al. (1988) } \\
\text { Foa et al. (1991) }\end{array}$ \\
\hline $\begin{array}{l}\text { Terapia cognitivo-conductual para el trastorno de ansiedad } \\
\text { generalizada }\end{array}$ & $\begin{array}{l}\text { Butler et al. (1991) } \\
\text { Borkovec et al. (1987) }\end{array}$ \\
\hline $\begin{array}{l}\text { Terapia de exposición y prevención de respuesta para el } \\
\text { trastorno obsesivo-compulsivo }\end{array}$ & Van Balkom et al. (1994) \\
\hline
\end{tabular}

Depresión

Terapia cognitiva para la depresión

Terapia conductual para la depresión

Dobson (1989)

Psicoterapia interpersonal para la depresión

Jacobson et al. (1996)

DiMascio et al. (1979)

Elkin et al. (1989)

Terapia dinámica breve

Gallangher-Thompson y Steffen (1994)

Trastornos sexuales y de pareja

Terapia de pareja conductual

Terapia de conducta para las disfunciones sexuales
Jacobson y Follette (1985)

LoPiccolo y Stock (1986)

Hurlbert et al. (1993)

Zimmer et al. (1987)

Tratamiento conductual-cognitivo con los ofensores sexuales

Marshall et al. (1991)

Nota: Las referencias bibliográficas correspondientes a esta tabla figuran en el Apéndice 1. 
Tabla 3. Tratamientos psicológicos efectivos (Chambless et al., 1996, modificado)

\begin{tabular}{|c|c|}
\hline TIPOS DE TRATAMIENTOS & EVIDENCIA EMPÍRICA \\
\hline \multicolumn{2}{|c|}{ Trastornos de la conducta alimentaria } \\
\hline Terapia cognitivo-conductual para la bulimia & $\begin{array}{l}\text { Agras et al. (1989) } \\
\text { Thackwray et al. (1993) }\end{array}$ \\
\hline Psicoterapia interpersonal para la bulimia & $\begin{array}{l}\text { Fairburn et al. (1993) } \\
\text { Wilfley et al. (1993) }\end{array}$ \\
\hline \multicolumn{2}{|l|}{ Trastornos adictivos } \\
\hline $\begin{array}{l}\text { Terapia cognitivo-conductual multicomponente para dejar } \\
\text { de fumar }\end{array}$ & $\begin{array}{l}\text { Hill et al. (1993) } \\
\text { Stevens y Hollis (1989) }\end{array}$ \\
\hline $\begin{array}{l}\text { Terapia cognitivo-conductual para la dependencia de cocaína } \\
\text { y de opiáceos }\end{array}$ & $\begin{array}{l}\text { Higgins et al. (1993) } \\
\text { Carroll et al. (1994) } \\
\text { Woody et al. }(1990)\end{array}$ \\
\hline Terapia dinámica breve para la dependencia de opiáceos & Woody et al. (1990) \\
\hline $\begin{array}{l}\text { Terapia de conducta multicomponente en el tratamiento del } \\
\text { alcoholismo }\end{array}$ & $\begin{array}{l}\text { Azrin (1976) } \\
\text { Drummomnd y Glautier (1994) } \\
\text { Eriksen et al. (1986) } \\
\text { O'Farrell et al. (1992) }\end{array}$ \\
\hline
\end{tabular}

Trastornos psicóticos

Programas de educación familiar para la esquizofrenia

Falloon et al. (1985)

Randolph et al. (1994)

Entrenamiento en habilidades sociales para mejorar la

Marder et al. (1996)

adaptación social en la esquizofrenia

Nota: Las referencias bibliográficas correspondientes a esta tabla figuran en el Apéndice 1.

Tabla 4.Tratamientos psicológicos efectivos (Chambless et al., 1996, modificado)

\begin{tabular}{|c|c|}
\hline TIPOS DE TRATAMIENTOS & EVIDENCIA EMPÍRICA \\
\hline \multicolumn{2}{|c|}{ Trastornos infantiles } \\
\hline Terapia de conducta para la enuresis & Houts et al. (1994) \\
\hline $\begin{array}{l}\text { Programa de entrenamiento para padres de niños con } \\
\text { problemas de conducta }\end{array}$ & $\begin{array}{l}\text { Walter y Gilmore (1973) } \\
\text { Wells y Egan (1988) }\end{array}$ \\
\hline Técnicas de control de hábitos inadaptativos & Azrin et al. (1980) \\
\hline Terapia cognitivo-conductual para niños ansiosos & $\begin{array}{l}\text { Kendall (1994) } \\
\text { Kendall et al. (1997) }\end{array}$ \\
\hline
\end{tabular}

Nota: Las referencias bibliográficas correspondientes a esta tabla figuran en el Apéndice 1.

1996), no deja de ser sorprendente que muchos psicólogos clínicos en ejercicio no estén adiestrados en este tipo de programas y que muchos programas de formación en psicología clínica se hagan a espaldas de estos conocimientos (Barlow y Hoffman, 1997).

Sin embargo, no se pueden disociar de los resultados obtenidos con estas tera- pias las características específicas de los terapeutas intervinientes. Cuando se constata en la clínica que algunos terapeutas, hagan lo que hagan, contribuyen a mejorar al paciente, mientras que en otros ocurre al contrario, conviene analizar las condiciones personales favorecedoras de la aplicación de un tratamiento efectivo. Una buena alianza terapéutica 
es condición necesaria, pero no suficiente, para un buen resultado terapéutico. Si bien se conoce poco al respecto, algunas características positivas del terapeuta son contar con sentido común, mostrar un equilibrio emocional y no ser rígido (adaptando con flexibilidad el modelo clínico al paciente, y no al revés), así como reflejar unas ganas genuinas de ayuda al paciente. En último término, se trata de hacer realidad un viejo aforismo en medicina: curar a veces; aliviar a menudo; consolar siempre.

\section{CONCLUSIONES}

La integración de las terapias psicológicas en el Sistema Nacional de Salud exige la evaluación rigurosa de las mismas y debe hacerse al hilo del aumento de la calidad de los servicios y de la reducción de costes (Barlow y Hoffman, 1997). Es más, la tendencia actual es a evaluar la eficacia de todos los tratamientos disponibles -psicológicos y psicofarmacológicos, solos o en combinación- en cada uno de los trastornos incluidos en el $D S M-I V$, así como los posibles efectos adversos generados por cada uno de ellos (Echeburúa, Corral y Salaberría, 1998; Elkin, 1994; Nathan y Gorman, 1998). De hecho, ya ha surgido una revista electrónica (In Session) que se ocupa de cuestiones clínicas relacionadas con la efectividad de los tratamientos, sean éstos psicológicos o psicofarmacológicos, en los distintos trastornos.

El informe del Task Force (1995), surgido desde fuera de la psicología clínica como una necesidad ineludible de evaluar lo que funciona en el ámbito de la salud, supone un punto de inflexión importante -y probablemente irreversible- en la evaluación de la eficacia de las terapias psicológicas. Más allá de las nebulosas psicoterapias basadas en la escucha, el apoyo y el inútil buceo en la desgraciada infancia del paciente, los protocolos de tratamiento en el marco de terapias breves (10/15 sesiones) y específicas tienden a imponerse, lo que no es incompatible con la necesaria flexibilidad en la aplicación de estos tratamientos en función de las peculiaridades específicas de cada paciente.

Según los estudios disponibles hasta la fecha, las terapias breves con pocas sesiones obtienen buenos resultados. De hecho, y al margen de la dificultad de aceptación de los tratamientos largos por parte de los pacientes (Pekarik, 1993), el alargamiento de una terapia no aumenta la utilidad o la eficacia de la misma (Bergin y Garfield, 1994). Incluso hay una probabilidad muy alta de que la intervención clínica sea ineficaz para un problema específico si el paciente no responde de forma satisfactoria tras las primeras sesiones (Echeburúa, 1993; Echeburúa, Corral, García y Borda, 1993).

Los desarrollos futuros, siempre al compás de la exigencia social de la evaluación, tienen que evolucionar en diversas direcciones. En primer lugar, demostrar que los tratamientos eficaces (beneficiosos para los pacientes en investigaciones clínicas controladas) son también efectivos (útiles en la práctica clínica habitual) y eficientes (de mayores beneficios y menores costes que otras terapias alternativas) (Turner, Beidel, Spaulding y Brown, 1995). Es decir, se trata de dar el paso de la investigación clínica a la práctica clínica habitual, que a veces es problemático y no está exento de diversos escollos (Sobell, 1996). $\mathrm{Y}$ en segundo lugar, asignar tratamientos distintos a tipos de pacientes específicos en el ámbito de un mismo trastorno -el denominado «emparejamiento» pacientetratamiento-, como ya se ha empezado a hacer en el estudio del alcoholismo ( $c f r$. Allen y Kaden, 1995).

La relación coste/beneficio no entraña un criterio meramente economicista. Se trata de determinar que los resultados 
justifican una inversión a nivel terapéutico (coste de la terapia), a nivel sanitario (ahorro de tratamientos ulteriores) y $a$ nivel social (menor absentismo laboral, menor uso de recursos sociales, prevención de problemas en otros miembros de la familia, etc.).

Hay una superioridad clara de la terapia cognitivo-conductual -una de las aportaciones más eficaces e ingeniosas del sentido común terapéutico- sobre otro tipo de tratamientos (terapia psicodinámica, terapia familiar, terapia humanista y terapia ecléctica) en una gran variedad de trastornos (Seligman, 1998). Algunas otras orientaciones clínicas (sistémicas, interpersonales, etc.) pueden ser a veces útiles en la medida en que incorporan técnicas procedentes del enfoque cognitivo-conductual. Ello no obsta para que haya cuadros clínicos, como las psicosis, los problemas somatomorfos y los trastornos de personalidad (a excepción quizá del tratamiento dialéctico conductual para el trastorno límite del grupo de Linehan, 1991), en que la terapia de conducta haya pinchado en hueso y los resultados obtenidos sean aún escasos.

El modelo conductual ha evolucionado hacia un planteamiento más cognitivo, integrando los estados afectivos y los procesos de pensamiento, pero conservando su original planteamiento científico (Cottraux, 1991). Respecto al inmediato futuro, los enfoques terapéuticos basados en la solución de problemas - un tema que puede ser común a distintas orientaciones- parecen desempeñar un papel importante en el desarrollo de los nuevos avances. De este modo, lo que puede explicar la eficacia similar de la terapia cognitiva y la terapia interpersonal en el tratamiento de la depresión es el acento puesto por una y otra en la estrategia de solución de problemas (Marks, 1992).

No es exagerado señalar que la necesidad de evaluación es una exigencia que la sociedad marca al psicólogo clínico y que la selección de las terapias eficaces debe estar sustentada en datos empíricos. La delimitación de las terapias breves, protocolizadas y efectivas constituye, sin duda, el mayor reto de futuro en este ámbito.

Una última reflexión se impone. Lo que es más efectivo en general - la terapia de conducta - no siempre está disponible. De hecho, los pacientes pueden acudir a un Centro de Salud Mental consultando por un determinado problema (una fobia social, un trastorno de pánico, una anorgasmia, etc.) en el que las técnicas conductuales constituyen la terapia de elección y recibir un tratamiento meramente farmacológico o psicológico de otra índole. Por chocante que pueda resultar, lo que parece hoy efectivo dista de estar disponible para la mayoría de la población. De este modo, hacer que lo útil sea utilizado se convierte en una prioridad de actuación.

\section{REFERENCIAS BIBLIOGRÁFICAS}

Allen, J.P., y Kadden, R.M. (1995). Matching clients to alcohol treatment. En R.K. Hester y W.R. Miller (Eds.), Handbook of alcoholism treatment approaches. Effective alternatives (2nd. ed.). Boston: Allyn and Bacon.

Baer, L., y Greist, J.H. (1997). An interactive computer-administered self-assessment and self-help program for behavior therapy. Journal of Clinical Psychiatry, 58, 2328.

Barlow, D.H. (1994). Psychological interventions in the era of managed competition. Clinical Psychology: Science and Practice, 1, 109-123.

Barlow, D.H. (1996). Health care policy, psychotherapy research, and the future of psychotherapy. Psychotherapy, 51, 1050-1058.

Barlow, D.H., y Hoffman, S.G. (1997). Efficacy and dissemination of psychological treatments. En D.M. Clark y C.G. Fairburn (Eds.), Science and practice of cognitive 
behavior therapy. Oxford: Oxford University Press.

Becoña, E. (1993). Programa para dejar de fumar. Santiago de Compostela: Servicio de Publicaciones de la Universidad.

Bergin, A.E., y Garfield, S.L. (Eds.) (1994). Handbook of psychotherapy and behavior change (4th. edition). New York: Wiley.

Brengelmann, J.C. (1977). Procedimientos de modificación de conducta: algunas tendencias recientes. Análisis y Modificación de Conducta, 4, 5-27.

Burgess, M., Gill, M., y Marks, I.M. (1998). Postal self-exposure treatment of recurrent nightmares: A controlled trial. British Journal of Psychiatry, 172, 257-262.

Caro, I. (Ed.) (1993). Psicoterapia e investigación de procesos. Valencia: Promolibro.

Chambless, D.L. (1996). In defense of dissemination of empirically supported psychological interventions. Clinical Psychology: Science and Practice, 3, 230-235.

Chambless, D.L., y Hollon, S.D. (1998). Defining empirically supported therapies. Journal of Consulting and Clinical Psychology, $66,3-18$.

Chambless, D.L., Sanderson, W.C., Shoham, V. et al. (1996). An update on empirically validated therapies. The Clinical Psychologist, 49, 5-18.

Cottraux, J. (1991). Terapias comportamentales y cognitivas. Barcelona: Masson.

Derogatis, L.R., y Wise, T.N. (1996). Trastornos depresivos y de ansiedad en la asistencia primaria. Barcelona: Martínez-Roca.

Echeburúa, E. (1993). Modificación de conducta y psicología clínica. Bilbao: Servicio de Publicaciones de la Universidad.

Echeburúa, E., Corral, P., y Salaberría, K. (1998). Terapia de conducta y tratamientos psicofarmacológicos. En M.A. Vallejo (Ed.), Manual de terapia de conducta (Vol. 1). Madrid: Dykinson.

Echeburúa, E., Corral, P., García, E., y Borda, M. (1993). Interactions between self-exposure and alprazolam in the treatment of agoraphobia without current panic: an exploratoy study. Behavioural and Cognitive Psychotherapy, 21, 219-238.

Elkin, J. (1994). The NIMH treatment of depression collaborative research programme: Where we began and where we are. En A.E. Bergin y S.L. Garfield (Eds.), Handbook of psychotherapy and behavior change (4th. edition). New York: Wiley.

Fernández-Montalvo, J., y Echeburúa, E. (1997). Manual práctico del juego patológico. Madrid: Pirámide.

Foa, E.B., Rothbaum, B.O., Riggs, D.S., y Murdock, T.B. (1991). Treatment of posttraumatic stress disorder in rape victims: A comparison between cognitive-behavioral procedures and counseling. Journal of Consulting and Clinical Psychology, 59, 715-723.

Foa, E.B., y Wilson, R. (1992). Venza sus obsesiones. Barcelona: Robinbook.

Garfield, S.L. (1996). Some problems associated with "validated" forms of psychotherapy. Clinical Psychology: Science and Practice, 3, 218-229.

Ginsberg, G., Marks, I.M., y Walters, H. (1984). Cost-benefit analysis of controlled trial of nurse therapy for neurosis in primary care. Psychological Medicine, 14, 683-690.

Greist, J.H., Marks, I.M., Baer, L., et al. (1998). Self-treatment for obsessive-compulsive disorder using a manual and a computerized telephone interview: A U.S-U.K. study. MD Computing, 15, 149-157.

Havik, O.E., y VanderBoss, G.R. (1996). Limitations of manualized psychotherapy for everyday practice. Clinical Psychology: Science and Practice, 3, 264-267.

Herink, R. (Ed.) (1980). The psychotherapy handbook: the $A$ to $Z$ guide to more than 250 different therapies use today. New York: New American Library.

Hersen, M., y Michelson, L. (1986). Issues in psychotherapy research. New York: Plenum Press.

Hickey, P. (1998). DSM and behavior therapy. The Behavior Therapist, March 1998, 4346.

Hickling, E.J., y Blanchard, E.B. (1997). The private practice psychologist and manualbased treatments: A case study in the treatment of post-traumatic stress disorder secondary to motor vehicle accidents. Behaviour Research and Therapy, 35, 191-203.

Labrador, F.J. (1992). El estrés: nuevas técnicas para su control. Madrid: Temas de Hoy. 
Labrador, F.J., y Castro, L. (1987). La adhesión al tratamiento médico: análisis y modificación. Revista Española de Terapia del Comportamiento, 2, 157-164.

Linehan, M.M., Arinstrong, H.E., Suárez, A., Allmon, D., y Heard, H.L. (1991). Cognitive behavioral treatment of chronically parasuicidal borderline patients. Archives of General Psychiatry, 48, 1060-1064.

Marks, I.M. (1981). Cure and care of neurosis. New York: Wiley and Sons (traducción, Martínez Roca, 1986).

Marks, I.M. (1991). Self-administered behavioural treatment. Behavioural Psychotherapy, 19, 42-46.

Marks, I.M. (1992). Behavioural psychotherapy towards the millenium. En J. Cottraux, P. Legeron y E. Mollard (Eds.), Which psychotherapies in year 2000? Amsterdam: Swets and Zeitlinger.

McAllister, A. (1976). TV as a medium for delivering behavior therapy. Stanford University: Institute for Communication Research.

McCrady, B.S., Rodríguez Villarino, R., y Otero-López, J.M. (1997). Los problemas de la bebida: un sistema de tratamiento paso a paso. Manual del terapeuta. Manual de autoayuda. Madrid: Pirámide.

McKenzy, N., Blanes, T., y Marks, I.M. (1995). Computerised clinical benefit-cost audit of mental health care II: Time input, costs, patient satisfaction. Journal of Mental Heath, 1, 71-78.

Nathan, P.E., y Gorman, J.M. (Eds.) (1998). A guide to treatments that work. Oxford: Oxford University Press.

Pekarik, G. (1993). Beyond effectiveness. Uses of consumer-oriented criteria in defining treatment success. En T.R. Giles (Ed.), Handbook of effective psychotherapy. New York: Plenum Press.

Pérez, M. (1996). Tratamientos psicológicos. Madrid: Universitas.

Salaberría, K., y Echeburúa, E. (1998). Longterm outcome of cognitive therapy's contribution to self-exposure in vivo to the treatment of generalized social phobia. Behavior Modification, 22, 262-284.

Seligman, M.E.P. (1995). The effectiveness of psychotherapy. The Consumer Reports Study. American Psychologist, 50, 965-974.
Seligman, M.E.P. (1998). Foreword-A purpose. En P.E. Nathan y J.M. Gorman (Eds.), $A$ guide to treatments that work. Oxford: Oxford University Press.

Sevillá, J., y Pastor, C. (1996). Tratamiento psicológico de la depresión. Un manual de autoayuda paso a paso. Valencia: Publicaciones del Centro de Terapia de Conducta.

Shapiro, D.A. (1996). «Validated» treatments and evidence-based psychological services. Clinical Psychology: Science and Practice, 3, 256-259.

Smith, M.L., Glass, G.V., y Miller, T.I. (1980). The benefits of psychotherapy. Baltimore, Maryland: John Hopkins University Press.

Sobell, L.C. (1996). Bridging the gap between scientists and practitioners: The challenge before us. Behavior Therapy, 27, 297-320.

Task Force on Promotion and Dissemination of Psychological Procedures (1995). Training and dissemination of empiricallyvalidated psychological treatments: Report and recommendations. The Clinical Psychologist, 48, 3-29.

Turner, S.M., Beidel, D.C., Spaulding, S.A., y Brown, J.M. (1995). The practice of behavior therapy: A national survey of cost and methods. The Behavior Therapist, 18, 1-4.

Wilson, G.T. (1996). Empirically validated treatments: Reality and resistence. Clinical Psychology: Science and Practice, 3, 241-244.

\section{APÉNDICE 1}

Agras, W.S., Schneider, J.A., Amow, B., Raebum, S.D., y Telch, C.F. (1989). Cognitivebehavioral and response-prevention treatments for bulimia nervosa. Journal of Consulting and Clinical Psychology, 57, 215-221.

Azrin, N.H. (1976). Improvements in the CR approach to alcoholism. Behaviour Research and Therapy, 14, 339-348.

Azrin, N.H., Nunn, R.G., y Frantz, S.E. (1980). Habit reversal vs. Negative practice treatment of nailbiting. Behaviour Research and Therapy, 18, 281-285.

Barlow, D.H., Craske, M.G., Cemy, J.A., y Klosko, J.S. (1989). Behavioral treatment of panic disorder. Behavior Therapy, 20, 261-282. 
Borkovec, T.D., Mathews, A.M., Chambers, A., Ebrahimi, S., Lytle, R., y Nelson, R. (1987). The effects of relaxation training with cognitive or nondirective therapy and the role of relaxation-induced anxiety in the treatment of generalizad anxiety. Journal of Consulting and Clinical Psychology, 55, 883-888.

Butler, G., Fennell, M., Robson, P., y Gelder, M. (1991). Comparison of behavior therapy and cognitive behavior therapy in the treatment of generalizad anxiety disorder. Journal of Consulting and Clinical Psychology, 59, 167-175.

Carroll, K.M., Rounsaville, B. J., Gordon, L.T., Nich, C., Jatlow, P., Bisighini, R.M., y Gawin, F.H. (1994). Psychotherapy and pharmacology for ambulatory cocaine abusers. Archives of General Psychiatry, 51, 177-187.

Clark, D.M., Salkovskis, P.M., Hackman, A., Middleton, H., Anastasiades, P., y Gelder, M. (1994). A comparison of cognitive therapy, applied relaxation, and imipramine in the treatment of panic disorder. British Journal of Psychiatry, 164, 759-769.

Di Mascio, A., Weissman, M.M., Prusoff, B.A., Neu, C., Zwilling, M., y Klerman, G.L. (1979). Differential symptom reduction by drugs and psychotherapy in acute depression. Archives of General Psychiatry, 36, 1450-2456.

Dobson, K.S. (1989). A meta-analysis of the efficacy of cognitive therapy for depression. Journal of Consulting and Clinical Psychology, 57, 414-419.

Drummond, D.C., y Glautier, S. (1994). A controlled trial of cue exposure treatinent in alcohol dependence. Journal of Consulting and Clinical Psychology, 62, 809-817.

Elkin, L., Shea, M.T., Watkins, J.T., Imber, S.D., Sotsky, S.M., Collins, J.F., Glass, D.R., Pilkonis, P.A., Leber, W.R., Docherty, J.P., Fiester, S.J., y Parloff, M.B. (1989). National Institute of Mental Health Treatment of Depression Collaborative Research Program: General effectiveness of treatments. Archives of General Psychiatry, 46, 971-982.

Eriksen, L., Bjomstad, S., y Gotestam, K.G. (1986). Social skills training in groups for alcoholics: One-year treatment outcome for groups and individuals. Addictive Behaviors, 11, 309-329.
Fairburn, C.G., Jones, R., Peveler, R.C., Hope, R.A., y O'Conner, M. (1993). Psychoteraphy and bulimia nervosa: Longer-term effects of interpersonal psychotherapy, behavior therapy, and cognitive behavior therapy. Archives of General Psychiatry, $50,419-428$.

Falloon, I.R.H., Boyd, J.L., McGill, C.W., Williamson, M., Razani, J., Moss, H.B., Gilderman, A.M., y Simpson, G.M. (1985). Family management in the prevention of morbidity of schizophrenia: Clinical outcome of a two year longitudinal study. Archives of General Psychiatry, 42, 887-896.

Foa, E.B., Rothbaum, B.O., Riggs, D.S., y Murdock, T.B. (1991). Treatment of posttraumatic stress disorder in rape victims: A comparison between cognitive-behavioral procedures and counseling. Journal of Consulting and Clinical Psychology, 59, 715-723.

Gallagher-Thompson, D., y Steffen, A.M. (1994). Comparative effects of cognitive behavioral and brief dynamic therapy for depressed family caregivers. Journal of Consulting and Clinical Psychology, 62, 543-549.

Heimberg, R.G., Dodge, C.S., Hope, D.A., Kennedy, C.R., y Zollo, L.J. (1990). Cognitive behavioral group treatment for social phobia: Comparison with a credible placebo control. Cognitive Therapy and Research, 14, 1-23.

Higgins, S.T., Budney, A.J., Bickel, W.K., Hughes, J.R., Foeg, F., y Badger, G. (1993). Achieving cocaine abstinence with a behavioral approach. American Journal of Psychiatry, 150, 763-769.

Hill, R.D., Rigdon, M., y Johnson, S. (1993). Behavioral smoking cessation treatment for older chronic smokers. Behavior Therapy, 24, 321-329.

Houts, A.C., Berman, J.S., y Abrainson, H. (1994). Effectiveness of psychological and pharmacological treatments for nocturnal enuresis. Journal of Consulting and Clinical Psychology, 62, 737-745.

Hurbelt, D.F., White, C.L., y Powel, R.D. (1993). Orgasm consistency training in the treatment of women reporting hypoactive sexual desire: An outcome comparison of women-only groups and couple-only 
groups. Journal of Behavior Therapy and Experimental Psychiatry, 24, 3-13.

Jacobson, N.S., Dobson, K.S., Truax, P.A., Addis, M.E., Koemer, K., Gollan, J.K., Gortner, E., y Prince, S.E. (1996). A component analysis of cognitive-behavioral treatment for depression. Journal of Consulting and Clinical Psychology, 62, 295-304.

Jacobson, N.S., y Follette, W.C. (1985). Clinical significance of improvement resulting from two behavioral marital therapy components. Behavior Therapy, 16, 249-262.

Kendall, P.C. (1994). Treating anxiety disorders in children: Results of a randomized clinical trial. Journal of Consulting and Clinical Psychology, 62, 100-110.

Kendall, P.C., Flannery-Schroeder, E., Panichelli-Mindel, S.M., Southam-Gerow, M., Henin, A., y Warman, M. (1997). Therapy for youths with anxiety disorders: A second randomized clinical trial. Journal of Consulting and Clinical Psychology, 65, 366-380.

LoPiccolo, J., y Stock, W.E. (1986). Treatment of sexual dysfunction. Journal of Consulting and Clinical Psychology, 54, 158-167.

Marder, S.R., Wirshing, W.C., Mintz, J., McKenzie, J., Johnston, K., Eckinan, T.A., Lebell, M., Zimmerman, K., y Lieberinan, R.P. (1996). Two-year outcome of social skills training and group psychotherapy for outpatients with schizophrenia. American Journal of Psychiatry, 153, 1585-1592.

Marshall, W.L., Jones, R., Ward, T., Johnston, P., y Barbaree, H.E. (1991). Treatment outcome with sex offenders. Clinical Psychology Review, 11, 465-485.

O'Farrell, T.J., Cutter, H.S.G., Choquette, K.A., Floyd, F.J., y Bayog, R.D. (1992). Behavioral marital therapy for male alcoholics: Marital and drinking adjustment during the two years after treatment. Behavior Therapy, 23, 529-549.

Öst, L.G., Salkovskis, P.M., y Hellstrom, K. (1991). One-session therapist-directed exposure vs. self exposure in the treatment of spider phobia. Behavior Therapy, 22, 407-422.

Randolph, E.T., Eth, S., Glynn, S., Paz, G.B., Leong, G.B., Shaner, A.L., Strachan, A., Van Vort, W., Escobar, J., y Liberina, R.P. (1994). Behavioural family management in schizophrenia: Outcome from a clinicbased intervention. British Journal of Psychiatry, 144, 501-506.

Stevens, V.J., y Hollis, J.F. (1989). Preventing smoking relapse, using an individually tailored skills-training technique. Journal of Consulting and Clinical Psychology, 57, 420-424.

Thackwray, D.E., Smith, M.C., Bodfish, J.W., y Meyers, A.W. (1993). A comparison of behavioral and cognitive-behavioral interventions for bulimia nervosa. Journal of Consulting and Clinical Psychology, 61, 639-645.

Trull, T.J., Nietzel, M.T., y Main, A. (1988). The use of meta-analysis to assess the clinical significance of behavior therapy for agoraphobia. Behavior Therapy, 19, 527-538.

Van Balkom, A.J.L.M., Van Oppen, P., Verineulen, A.W.A., Nauta, N.C.E., Vorst, H.C.M., y Van Dyck, R. (1994). A metaanalysis on the treatment of obsessive compulsive disorder: A comparison of antidepressants, behaviour and cognitive therapy. Clinical Psychology Review, 14, 359-381.

Walter, H.I., y Gilmore, S.K. (1973). Placebo versus social learning effects in parent training procedures designed to alter the behavior of aggresive boys. Behavior Therapy, 4, 361-377.

Wells, K.C., y Egan, J. (1988). Social leaming and systems farnily therapy for childhood oppositional disorder: Comparative treatment outcome. Comprehensive Psychiatry, 29, 138-146.

Wilfley, D.E., Agras, W.S., Telch, C.F., Rossiter, E.M., Schneider, J.A., Cole, A.G., Sifford, L., y Raeburn, S.D. (1993). Group cognitive-behavioral therapy and group interpersonal psychotherapy for the nonpurging bulimic individual: A controlled comparison. Journal of Consulting and Clinical Psychology, 61, 296-305.

Woody, G.E., Luborsky, L., McLellan, A.T., y O'Brien, C.P. (1990). Corrections and revised analyses for psychotherapy in methadone maintenance patients. Archives of General Psychiatry, 47, 788-789.

Zimmer, D. (1987). Does marital therapy enhance the effectiveness of treatment for sexual dysfunction? Journal of Sex and Marital Therapy, 13, 193-209. 